bad became particularly important during this time, for example in relation to a series of incidents involving the emergency home treatment team.

5. Finally, staff needs have remained an enduring theme throughout, not surprisingly since bad clinical outcomes are stressful for the professionals involved. Issues such as access to psychological help, security arrangements, and the way the organisation responds to wilful assaults on staff give important signals to the workforce, and all have been improved over the years. But perhaps the most influential factor is the staff's perception of whether the organisation fosters a no blame culture when conducting serious incident inquiries. This is hard to measure, and is obviously highly subjective, but may have a profound effect on morale if the balance is wrong. The perceived culture of the organisation has been a recurrent topic for discussion at peer review meetings, and although there is an inevitable tension around this issue (after all, the organisation must hold its staff to account for their actions), certain characteristics of the meeting encourage transparency in a way that makes it harder for the organisation to be seen as blaming if it in fact isn't. These include the presence of senior executives at meetings, and the presentation of inquiry outcomes by their authors.

\section{Constraints and difficulties}

Not all bad clinical outcomes can be put down to system problems. Individuals can make serious mistakes, or be negligent, and need to be held accountable. In these situations, particularly if there appear to be no wider lessons to be learned, an incident may be less suitable for a peer group approach, or perhaps may need to be looked at anonymously. For the aim is not to have a witch-hunt, but to look at bad outcomes in an atmosphere that fosters trust, openness, and selfreflection, but does not shy away from asking penetrating questions.

\section{Conclusion}

A long-established peer group serious incident review has adjusted to recent NHS changes, with its importance, if anything, growing over time. This is partly because it enables as many clinical staff as possible to contribute to the organisation's thinking about risk management, and partly because it serves as an effective educational tool. This latter role will become of ever greater importance as the education agenda of Modernising Medical Careers takes effect, with its emphasis on self-reflectivity and learning within the clinical context. In fact, one of the challenges for all health systems trying to improve clinical care is to design effective structures and feedback loops for learning from bad outcomes, and for these to become deeply embedded within an organisation's learning culture. It is hard to envisage peer reviews not playing a key part of this learning and governance philosophy in the years ahead.

\section{Declaration of interest}

None.

\section{References}

ANDERSEN, B., \& FAGERHAUG, T. (2000) Root Cause Analysis: Simplified Tools and Techniques. ASQ Quality Press.

APPLEBY, L. (2006) Avoidable Deaths. Five-Year Report of the National Confidential Inquiry into Suicide and Homicide by People with Mental IIIness. Department of Health.

NEAL, L. A. WATSON, D. HICKS, T, et a (2004) Root cause analysis applied to the investigation of serious untoward incidents in mental health services. Psychiatric Bulletin, 28, 75-77.

ROSE, N. (2000) Six years' experience in Oxford: review of serious incidents. Psychiatric Bulletin, 24, 243-246. SALTER, M. (2003) Serious incident inquiries: a survival kit for psychiatrists. Psychiatric Bulletin, 27, $245-247$

VINCENT, C., STANHOPE, N. \& TAYLORADAMS, S. (2000) Developing a systematic method of analysing serious incidents in mental health. Journal of Mental Health, 9, 89-103.

Nick Rose formerly Consultant Psychiatrist and Honorary Senior Lecturer, University of Oxford, c/oWarneford Hospital, Old Road, Headington, Oxford OX37JX, email: nick.rose276@ntlworld.com

\title{
Help is at hand for people bereaved by suicide and other traumatic death
}

Bereavement following suicide is traumatic. Guilt, shame, stigma and feelings of rejection and isolation set it apart from the sadness following other kinds of death and may make it difficult for the bereaved person to obtain help (Harwood et al, 2002; Hawton \& Simkin, 2003; Beautrais, 2004). The necessary official processes surrounding death by suicide, like the police and coroner's investigations, can add to the trauma (Biddle, 2003). This may be compounded by inaccurate or insensitive media reporting. Bereaved individuals are at risk of increased morbidity from abnormal grief reactions (Mitchell et al, 2005) and suicide (Qin et al, 2002), and they often need considerable support (de Groot et al, 2006; 2007).

Bereavement through suicide is not uncommon. There are about 5500 deaths by suicide each year in the UK and it is suggested that on average 6 people are deeply affected by each one, which amounts to at least 30000 bereaved individuals per year. If we take into 
(8)

special articles account also those outside of the immediate family, like colleagues, school friends or professionals, the number grows. Despite this, many of us, in both personal and professional roles, may not know how best to deal with the grief of those bereaved. Moreover, the specific nature of bereavement by suicide often makes it difficult for bereaved individuals to seek help. Also, until recently, few resources were readily available (Andriessen et al, 2007). Self-help groups are one means of getting support (Andriessen \& Farberow, 2002), but not all people will want to use this form of help or indeed are aware of it. Another potential source of support is through written material (Wertheimer, 2001). This article describes the development, content and potential uses of new resources developed by the University of Oxford in collaboration with the Department of Health.

\section{Developing the resources}

The Department of Health's suicide prevention strategy for England (2002) has as one of its goals the promotion of mental well-being in the wider population. Targeting people bereaved by suicide, the Department of Health established a stakeholder group and commissioned the Centre for Suicide Research at Oxford University to develop the resources. This built on a bereavement information pack by the Centre for Suicide Research (Hill et al, 1997).

The stakeholder group included national bereavement organisations and key agencies (Box 1). Several members of the group had been bereaved by suicide themselves. In addition, specific advice was sought - for example, on the needs of children, young people, and black and minority ethnic groups. Representatives of various faith groups were also consulted.

Research suggests that people bereaved by suicide want information about the death and suicide more generally, about the process of mourning, on practical and administrative issues and how to access support. They also want support in the workplace and better awareness of their needs among professionals and

\section{Box 1. Key stakeholders included in the planning} process

- Survivors of Bereavement by Suicide

- Samaritans

- Cruse Bereavement Care

- Compassionate Friends

- PAPYRUS, Prevention of Young Suicide

- Loss Group, Leeds

- Hospital chaplain

- Railway Safety and Standards Board

- Home Office coroners (now at the Department for Constitutional Affairs)

- Care Services Improvement Partnership

- Safer Custody Group

- Coroners Officers Association

- The Coroners' Society of England and Wales

- National Association of Funeral Directors

- Metropolitan Police

- ZitoTrust service providers (Clark \& Goldney, 2000; Grad et al, 2004). This was borne out by working with the stakeholders who had direct experience of being bereaved. We decided to produce a number of resources:

- a bereavement booklet covering the issues important for bereaved individuals;

- a postcard for professionals to raise awareness of the issue and the availability of the new resources;

- a wallet card to be given to bereaved people at first contact with services, with the telephone number to the National Health Service (NHS) helpline (NHS Direct).

It was clear that many of the issues that needed to be covered were of relevance to a broader group of bereaved individuals, including those traumatically bereaved by death other than by suicide (in some cases it may be clear only after the coroner's inquest, which may not take place for several months, whether the death was by suicide or not). The design and the name of the booklet had to pitch the tone between an understanding of the despair and the need to engender hope.

\section{Help is at Hand}

The 48-page booklet is divided into the following sections:

1. Introduction

2. Practical matters - discusses issues such as arranging the funeral, dealing with the will and the estate. There is a checklist of the organisations that should be notified about the death and a form that can be copied and sent to the various organisations. More specific issues such as the police investigation, the coroner's inquest and media reporting are also covered. The booklet gives information about these procedures and suggests ways to deal with them.

3. Experiencing bereavement - outlines current theories of bereavement and offers suggestions on how to cope with the various aspects of grief. It describes feelings and emotions which may be experienced, including those aspects of bereavement which may be more intense for people bereaved by suicide, such as unpleasant recurring images, constant searching for the reasons behind the death, guilt, abandonment and rejection. There is guidance for the bereaved on what to say to others about the death and what to do if they themselves experience suicidal feelings.

4. Bereaved people with particular needs - looks at the specific needs of parents who have lost a child, the needs of children, young people, older people, homosexual and bisexual people, and people with learning disabilities.

5. How friends and colleagues can help - advice for friends, employers, work colleagues, teachers, and prison, police, health and social care personnel.

6. Sources of support - information on dedicated bereavement organisations, self-help groups, religious, spirituality and faith groups, counselling, homosexual support agencies, services for the elderly and specific ethnic groups. Useful websites are also listed. 


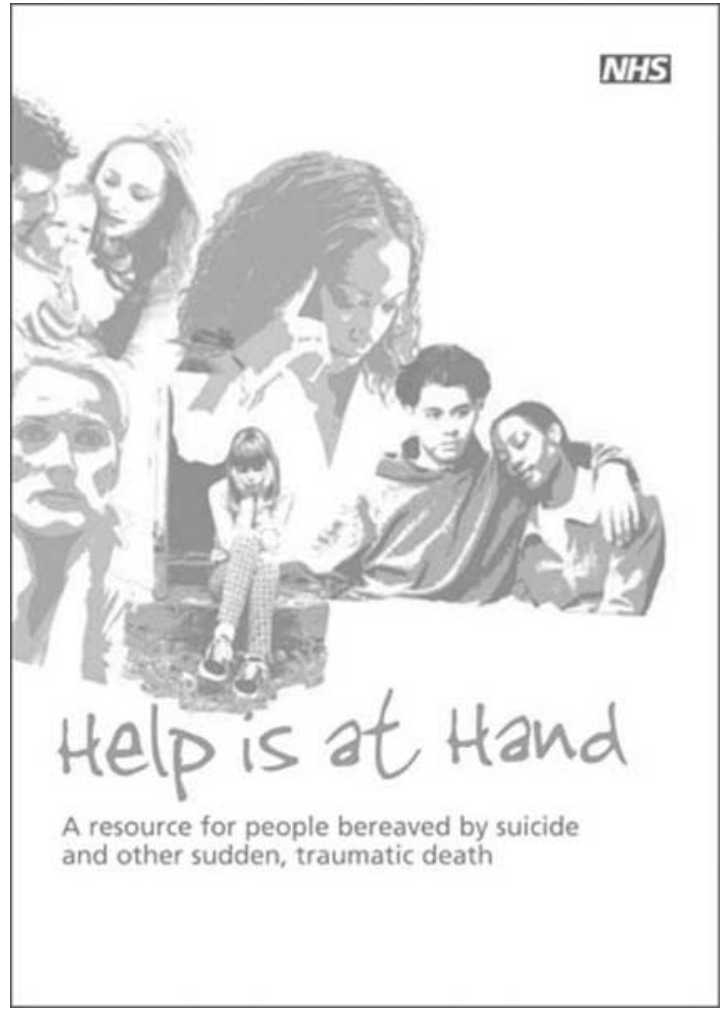

Various books, audiotapes, videotapes and DVDs on bereavement in general are listed, some specifically for parents and children. Resources on bereavement by suicide and on suicide itself, including self-help books and personal accounts of loss through suicide are also provided. There is also guidance on resources for professionals.

\section{How to use the resources}

The booklet is intended for people bereaved by suicide, those who can support them (e.g. family, friends and colleagues), and professionals dealing with bereaved individuals. It could also serve as a useful educational resource. It has been endorsed by the organisations involved with its development, including the Royal College of Psychiatrists.

The postcard for professionals highlights the problems and needs of people bereaved by suicide and professionals. The wallet card has been sent to organisations likely to have first contact with the bereaved, for example medical staff, coroners and their investigators, the police, and NHS Direct. We hope this article will raise awareness of the resources among psychiatrists.

\section{Declaration of interest}

None. Funding detailed in Acknowledgements.

\section{Acknowledgements}

We thank all members of the stakeholder group for their extensive input to the development of Help is at Hand, Philip Tierney (Foundation Design Consultants) for his contribution to the design of the booklet, and the Department of Health and Oxfordshire and Buckinghamshire Mental Health NHS Foundation Trust for supporting this work.

Some content of this report is based, with permission of Hogrefe \& Huber, on a report published in Crisis (Andriessen et al, 2007).

The booklet can be downloaded from the Department of Health's website (http://www.dh.gov.uk/en/ Publicationsandstatistics/Publications/PublicationsPolicy AndGuidance/DH_4139006). Hard copies can be ordered free of charge (Quote 274206/ Help is at Hand) from: Department of Health Publications Orderline, PO Box 777, London SE1 6XH, tel: 087015554 55, fax: 016237245 24, or by emailing dh@prolog.uk.com.

Help is at Hand was highly commended in the 2007 BMA Patient Information (Printed Materials) Awards.

\section{References}

ANDRIESSEN, K. \& FARBEROW, N. L. (eds) (2002) European Directory of Suicide Survivor Services. International Association for Suicide Prevention (http://www.med.uio.no/iasp/files/ europdir_text.pdf)

ANDRIESSEN, K., BEAUTRAIS, A. GRAD, O. T., et al (2007) Current understandings of suicide survivor issues: research, practice, and plans. Report of the 1st International Suicide Postvention Seminar, September 8 2006, Portoroz, Slovenia. Crisis: The Journal of Crisis Intervention and Suicide Prevention, 28, 211-213.

BEAUTRAIS, A. L. (2004) Suicide Postvention. Support for Families, Whānau and Significant Others After a Suicide. A Literature Review and Synthesis of Evidence. Ministry of Youth Affairs New Zealand.

BIDDLE, L. (2003) Public hazards or private tragedies? An exploratory study of the effect of coroners' procedures on those bereaved by suicide. Social Science and Medicine, 56, 1033-1045.

CLARK, S. E. \& GOLDNEY, R. (2000) The impact of suicide on relatives and friends. InThe International Handbook of Suicide and Attempted Suicide (eds K. Hawton \& K. Van Heeringen). pp. 467-484. Wiley.

DE GROOT, M. H., DE KEIJSER, J. \& NEELEMAN, J. (2006) Grief shortly after suicide and natural death: a

comparative study among spouses and first-degree relatives. Suicide and LifeThreatening Behavior, 36, 418-431.

DE GROOT, M., DE KEIJSER, J., NEELEMAN, J., et al (2007) Cognitivebehavioural therapy to prevent complicated grief among relatives and

spouses bereaved by suicide. A cluste randomised controlled trial. BMJ, 334, 994-996.

DEPARTMENT OF HEALTH (2002)

National Suicide Prevention Strategy for England. Department of Health.

GRAD, O., CLARK, S., DYREGROV, K., et al (2004) What helps and what hinders the process of surviving the suicide of somebody close? Crisis: The Journal of Crisis Intervention and Suicide Prevention, 25, 134-139.

HARWOOD, D., HAWTON, K., HOPE, T., et al (2002) The grief experiences and needs of bereaved relatives and friends of older people dying through suicide: a descriptive and case-control study. Journal of Affective Disorders, 72 , 185-194.

HAWTON, K. \& SIMKIN, S. (2003) Helping people bereaved by suicide. Their needs require special attention. BMJ, 327, 177-178.

HILL, K., HAWTON, K., MALMBERG, A et al (1997) Bereavement Information Pack: ForThose Bereaved by Suicide or Other Sudden Death. Gaskell.

MITCHELL, A. M., KIM,Y., PRIGERSON, H. G., et al (2005) Complicated grief and suicidal ideation in adult survivors of suicide. Suicide and Life-Threatening Behavior, 35, 498-506.

QIN, P., AGERBO, E. \& MORTENSEN, P. B. (2002) Suicide risk in relation to family history of completed suicide and psychiatric disorders: a nested case-control study based on longitudinal registers. Lancet, 360, $1126-1130$.

WERTHEIMER, A. (2001) A Special Scar (2nd ed). Brunner-Routledge.

*Keith Hawton Professor of Psychiatry and Director, Centre for Suicide Research, Oxford University, Department of Psychiatry, Warneford Hospital, Headington, Oxford OX37JX, email: keith.hawton@psych.ox.ac.uk, Sue Simkin Researcher and Coordinator, Centre for Suicide Research, Oxford University, Sian Rees Senior PolicyAdviser, Department of Health 